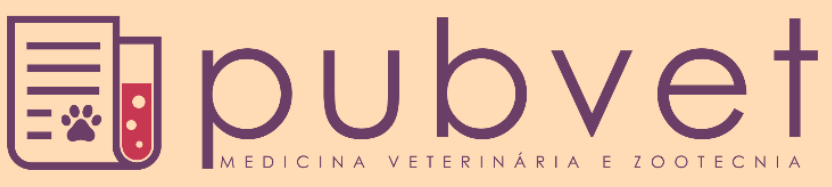

https://doi.org/10.31533/pubvet.v13n2a269.1-7

\title{
Tempo para recuperação da atividade ovariana luteal cíclica em vacas mestiças leiteiras magras com anestro
}

\author{
Ademir de Moraes Ferreira ${ }^{1 *} \bullet$, Isis Lustosa Goulart ${ }^{2} \theta$, José Rogério Moura de Almeida Neto ${ }^{2} \theta$ \\ ${ }^{I}$ Doutores, Professores da Fundação Dom André Arcoverde (FAA), Faculdade de Medicina Veterinária de Valença \\ *Autor para correspondência, E-mail:ademirferreira9@yahoo.com.br
}

Resumo. $\mathrm{O}$ experimento teve como objetivo conhecer o tempo (dias) necessário para o restabelecimento da atividade ovariana luteal cíclica (AOLC) em vacas mestiça Holandês $\mathrm{x}$ Zebu (HZ) não lactante, magras e em anestro com ovários inativos. Doze vacas girolando, com peso médio de 315,0 $\pm 29,4 \mathrm{~kg}$ (magras), foram alimentadas com volumoso e concentrado para ganho de peso até que a AOLC fosse reiniciada (volta do cio). A cada sete dias os animais foram pesados e tiveram o sangue coletado para dosagem de progesterona (RIA). A AOLC foi avaliada pela concentração de progesterona no soro sanguíneo, pelo exame dos ovários por palpação retal e observação visual do estro. A recuperação da AOLC ocorreu em 114,0 \pm 37,9 dias, após um ganho de peso total médio de 77,7 $\pm 11,2 \mathrm{~kg}$ /animal, que corresponde a 0,68 $\mathrm{kg} / \mathrm{dia}$. No grupo controle, seis animais receberam alimentação de mantença para o baixo peso apresentado e permaneceram em anestro durante todo o período experimental. A demora na recuperação da AOLC em vacas mestiças HZ, magras e com anestro, mostra como é indesejável permitir que o animal adquira a condição de anestro por subnutrição, por alongar o período de serviço e, em consequência, o intervalo de partos (menos leite e menor número de bezerros), com reflexo negativo no desempenho econômico da atividade leiteira.

Palavras Chaves: bovino, recuperação anestro, tempo (dias)

\section{Anestrus recovering time in cross bred milk cows thin}

Abstract. The aim of this experiment was to determine the time required for the reestablishment of the ovarian luteal cyclic activity (OLCA) in dry crossbred Holstein-Zebu (HZ) cows, thin and in anestrus with inactive ovaries. Twelve animals with an average weight of $315.0 \pm 29.4 \mathrm{~kg}$, were fed with forage and concentrate, in order to gain weight until reestablishment of OLCA. Blood samples for Progesterone analysis and weight measures of the animals were taken weekly. Estrous cycles onset was determined by plasma P4 level > 1.0 $\mathrm{ng} / \mathrm{ml}$, by ovarian rectal palpation and visual estrus detection. The OLCA recovery time was $114.0 \pm 37.9$ days, after an overall weight gain of $77.7 \pm 11.2 \mathrm{~kg} /$ animal, which is equivalent a $0.68 \mathrm{~kg} /$ day gain. The six control animals received a maintenance diet for the low weight and remained in anestrus during the experiment. The delay in the OLCA recovery time in crossbred $\mathrm{HZ}$ cows, thin and in anestrus shows how is undesirable to allow the animal to get in anestrus by sub nutrition. There is increase on the service time as well as on the parturition interval, with a negative impact on the economic performance of the dairy activity.

Keywords: Anestrus recover, cattle, crossbred, sub nutrition, time 


\title{
Tiempo para recuperación de la actividad ovárica luteal cíclica en vacas mestizas lecheras magras con anestro
}

\begin{abstract}
Resumen. El experimento tuvo como objetivo conocer el tiempo (días) necesario para el restablecimiento de la actividad ovárica luteal cíclica (AOLC) en vacas mestiza Holstein $\mathrm{x}$ Cebú (HZ) no lactante, magras y en anestro con ovarios inactivos. Doce vacas Girolando, con un peso medio de $315,0 \pm 29,4 \mathrm{~kg}$ (magras), fueron alimentadas con voluminosos y concentrados para ganancia de peso hasta que la AOLC fuera reiniciada (vuelta del celo). A cada siete días los animales fueron pesados y tuvieron la sangre recogida para la dosificación de progesterona (RIA). La AOLC fue evaluada por la concentración de progesterona en el suero sanguíneo, por el examen de los ovarios por palpación rectal y observación visual del estro. La recuperación de la AOLC ocurrió en 114,0 \pm 37,9 días, después de una ganancia de peso total promedio de 77,7 $\pm 11,2 \mathrm{~kg} / \mathrm{animal}$, que corresponde a $0,68 \mathrm{~kg} / \mathrm{día}$. En el grupo control, seis animales recibieron alimentación de mantenimiento para el bajo peso presentado y permanecieron en anestro durante todo el período experimental. La demora en la recuperación de AOLC en vacas mestizas HZ, magras y con anestro, muestra cómo es indeseable permitir que el animal adquiera la condición de anestro por desnutrición, por alargar el período de servicio y, en consecuencia, el intervalo de partos (menos leche y menor número de terneros), con reflejo negativo en el desempeño económico de la actividad lechera.
\end{abstract}

Palabras claves: bovino, recuperación anestro, tiempo (días)

\section{Introdução}

A cadeia agroindustrial do leite é reconhecida como uma das mais importantes do agronegócio nacional sob a ótica social e econômica, estando presente em todo o território nacional com papel relevante no suprimento de alimentos, na geração de empregos e de renda para a população. Neste contexto, a agropecuária vem impulsionando o crescimento do Produto Interno Bruto (PIB) do país, representando quase 25\% do PIB Nacional em 2012 (ANUALPEC, 2017). O Brasil possui o maior rebanho bovino comercial do mundo, contando com aproximadamente 212 milhões de animais, dos quais quase 23 milhões são vacas leiteiras, pertencentes a 1,3 milhões de produtores. Estas matrizes produziram cerca de 32 bilhões de litros de leite em 2012, colocando o país em terceiro lugar no ranking mundial de produção (ANUALPEC, 2017). Contudo, a pecuária leiteira nacional ainda é caracterizada pela baixa produtividade dos rebanhos, visto que o aumento do volume de leite produzido ao longo dos anos ocorreu, em grande parte, devido ao aumento do número de vacas ordenhadas e não por melhoria na produtividade, embora esta tenha tido um pequeno aumento na última década.

Uma das limitações para o aumento da produtividade no rebanho leiteiro nacional é o fraco desempenho reprodutivo, traduzido pela baixa taxa de nascimento devida, principalmente, ao prolongado anestro pósparto (Hess et al., 2005), provocado, na maioria das vezes por severo balanço energético negativo (Grummer et al., 2010), pela ação da subnutrição sobre a atividade ovariana. Anestro (ausência do cio) é a condição de aciclicidade ovariana, sendo caracterizado por completa inatividade sexual, falta de manifestação de cio e anovulação, acompanhado da concentração de progesterona no sangue $<1,0 \mathrm{ng} / \mathrm{ml}$ (Montiel \& Ahuja, 2005).

No Brasil é alta a taxa de vacas em anestro até 90 dias pós-parto, bem como acima desse período (Ferreira et al., 1992), consequência tanto da insuficiente ingestão de alimento, como da qualidade inferior da dieta, uma vez que prolongada restrição de dieta energética induz o anestro em vacas (Beam \& Butler, 1999; Schillo, 1992; Vanholder et al., 2005). A duração do anestro é influenciada por muitos fatores, como estímulo da amamentação, nível de nutrição, condição corporal, raça e idade da vaca, presença do touro, estação do ano, etc., e esses fatores podendo atuar isoladamente ou em associação (Short et al., 1990; Stagg et al., 1998; Tervit et al., 1977; Wiltbank et al., 2002). O reinício da atividade luteal cíclica (AOLC) seguinte ao anestro por severa restrição alimentar pode demorar várias semanas (Ferguson, 1996). Rhodes et al. 
(1995) citam que a duração do período de recuperação do anestro, até o reinício da AOLC, foi inversamente relacionada à duração do período de perda de peso até o animal entrar em anestro, ou seja, quanto mais tempo a vaca perder peso e permanecer magra, mais tempo a vaca levará para apresentar estro após passar a receber uma alimentação calculada para ganho de peso. A grande importância econômica do anestro é prolongar o intervalo de partos, diminuindo o número de crias e de lactações durante a vida útil do animal, pois modelos econômicos indicam que o rendimento máximo é obtido quando as vacas parem a cada 11 a 13 meses (Ferguson, 1996; Stevenson, 1996a, 1996b).

Peter et al. (2009) classificam o anestro em quatro tipos (I, II, III e IV) com base em três estágios funcionais do desenvolvimento folicular (emergência, divergência e ovulação), ou seja, de acordo com a dinâmica folicular e luteal ovariana do animal. A quase totalidade do anestro predominante em rebanhos leiteiros brasileiros coincide com o tipo I da citada classificação (Ferreira, 2010, 2012), caracterizado por folículos que iniciam seu crescimento e chegam ao máximo até a emergência (4 mm de diâmetro), portanto, sem divergência ou estabelecimento de um folículo dominante (FD), sendo descritos como ovários inativos (Drion et al., 2000). Pereira et al. (2010) relataram em vacas magras folículos no máximo com 6,5mm de diâmetro. Esta é uma condição presente em casos de extrema subnutrição, doenças debilitantes ou em curto período pós-parto. Adicionalmente, em dois exames ultrassonográficos com intervalo de sete dias, nenhuma alteração é observada nas estruturas ovarianas, acompanhado pela ausência característica de CL ou estruturas císticas. Peter et al. (2009) afirmam ainda que vacas no início do pós-parto ou com baixa reserva corporal de gordura (causas do anestro tipo I) não respondem a tratamento hormonal, e preferem focar o aumento da fertilidade do animal ou rebanho promovendo um bom manejo (corrigindo o manejo nutricional), sendo isto mais apropriado que contar com o difundido uso de hormônios exógenos. Por ser este o tipo de anestro que predomina no Brasil, provocado por subnutrição que emagrece os animais, é que não se podem extrapolar para o Brasil os resultados positivos obtidos em países com melhor condição de manejo nutricional, onde os tratamentos hormonais para anestro induzem a ciclicidade em muitas vacas. Isto ocorre porque a quase totalidade do anestro nestes países desenvolvidos está relacionada a animais com melhor condição corporal e presença de folículos maiores, mas que tão somente ainda não ovularam, conforme citado por vários autores na revisão efetuada por (Ferreira, 2010, 2012).

A proposta do presente trabalho é de conhecer o tempo (dias) para restabelecimento da AOLC em vacas mestiça Holandês x Zebu, magras e com anestro, após serem devidamente alimentadas para ganho de peso.

\section{Material e métodos}

Os trabalhos foram conduzidos no CEPARB (Centro de Estudo e Pesquisa Aplicada em Reprodução Bovina) da Faculdade de Medicina Veterinária de Valença, Fundação Dom André Arcoverde, município de Valença, Estado do Rio de Janeiro. Foram utilizadas 18 vacas adultas, mestiças Holandesas x Zebus, com peso médio de 315,0, $\pm 29,4 \mathrm{~kg}$ (magras), não lactantes, com ausência de atividade ovariana luteal cíclica (AOLC), ou seja, em anestro, constatado pela concentração de progesterona no soro sanguíneo abaixo de $1,0 \mathrm{ng} / \mathrm{ml}$ (Mapletoft et al., 2000; Wiltbank et al., 1990), por três semanas consecutivas, e por dois exames ginecológicos com intervalo de 12 dias. Esses animais adquiriram a condição de anestro após restrição alimentar até cessação da AOLC, e continuaram perdendo peso por mais 30 a 45 dias, quando foram distribuídos em dois grupos que receberam:

A) Dieta de mantença (grupo controle): seis $(n=6)$ animais.

B) Dieta para ganho de peso: doze $(n=12)$ animais até que os ovários reiniciassem sua atividade luteal cíclica (grupo tratado). A alimentação constou de silagem de capim-elefante (Napier) e concentrado contendo, respectivamente, $22,9 \%$ e $89,0 \%$ de matéria seca, com $5,6 \%$ e $11,0 \%$ de proteína bruta e $53,0 \%$ e $64,0 \%$ de NDT.

A AOLC foi avaliada como segue: a) exame ginecológico a cada 12 dias, por palpação retal, verificandose a presença de corpo lúteo nos ovários; b) observação visual do estro três vezes ao dia (manhã, meio dia 
e entardecer), com um mínimo de 30 minutos cada; c) níveis de progesterona no soro sanguíneo a cada sete dias.

Com objetivo de verificar a influência de alguns fatores sobre o tempo (dias) para o reinicio da AOLC em vacas de condição corporal inferior, foi calculada a correlação de Pearson para as variáveis: peso inicial, peso final, ganho de peso (kg e \%), ganho de peso/dia e tempo para reinicio da AOLC. A relação entre o tempo (número de dias) para recuperação da AOLC e ganho de peso médio diário dos animais foi avaliada por análise de regressão pelo método dos mínimos quadrados.

\section{Resultados e discussão}

Vacas mestiças Holandesas x Zebus, não lactante, magras e com anestro por ovários inativos, recuperaram a AOLC em 114,0 \pm 37,9 dias (16,3 semanas) após o início da alimentação que proporcionou um ganho de peso médio diário de $0,68 \mathrm{~kg} /$ dia. Os seis animais do grupo controle (magras), que receberam dieta de mantença para o baixo peso apresentado, permaneceram em anestro ou sem AOLC durante o período experimental. O tempo necessário para recuperação da AOLC em bovinos com anestro, devido a ovários inativos, foi estudado em vacas não lactantes de $320 \mathrm{~kg}$ (Hale, 1975), em novilhas Angus x Hereford de $320 \mathrm{~kg}$ (Imakawa et al., 1986), em vacas Hereford não lactantes de $319 \mathrm{~kg}$ (Richards et al., 1986) e em novilhas da raça Brahmas com $307 \mathrm{~kg}$ (Rhodes et al., 1995), com o retorno da AOLC ocorrendo, respectivamente, em 105, 49, 63 e 54,5 dias, com ganhos de peso vivo de 0,460 kg; 1,800 kg; 0,803 kg e $1,32 \mathrm{~kg} /$ dia.

No presente trabalho o tempo necessário para recuperação da AOLC foi de 114,0 \pm 37,9 dias, resultado acima daqueles obtidos pelos autores citados. A grande variação nas respostas pode ser explicada pelas diferentes raças, grau de sangue e categoria (novilhas ou vacas) dos animais, pelo maior ou menor peso inicial e condição corporal, e pelos diferentes níveis nutricionais usados nos vários experimentos. Esses fatores podem ter influenciado a taxa de ganho de peso/dia. A taxa de ganho depende do tempo para reinício da AOLC. Maior ganho de peso/dia reduz o tempo (número de dias) para recuperação da AOLC ( $\mathrm{r}=-0,90$; $\mathrm{P}<0,05)$, o que está de acordo com os trabalhos de Hale (1975), Imakawa et al. (1986), Richards et al. (1986) e Rhodes et al. (1995).

Ovários de vacas magras sem AOLC apresentam-se pequenos, duros e lisos à palpação retal, ou de tamanho e consistência normais, sem atividade de crescimento folicular normal, condições não mencionadas nos diferentes experimentos citados, mas capazes de afetar o tempo requerido para retorno da OLCA. Almeida Neto (2013) examinou, usando ultrassom, vacas mestiças leiteiras, magras e com anestro, e encontrou a maioria com folículos pequenos $(<4 \mathrm{~mm})$ ou não visíveis, e raros animais com folículos médios no máximo com $\leq 6,1 \pm 0,7$ de diâmetro, o que está de acordo com o anestro tipo I mencionado por Peter et al. (2009) e com as observações de Gwazdauskas et al. (2000), segundo os quais uma prolongada restrição energética na dieta reduz a secreção de hormônios ovarianos e o desenvolvimento folicular, bem como predominam folículos com $<5 \mathrm{~mm}$ nos ovários de vacas alimentadas com dieta de baixa energia (severamente subnutridas). Estas condições não são mencionadas ou detalhadas nos diversos experimentos, mas são capazes de influenciar no tempo de recuperação da AOLC.

Dos 12 animais em anestro utilizados no presente experimento, apenas três apresentavam ovários pequenos, duros e lisos à palpação retal, consequência, em geral, de longo período de subnutrição (Butler, 1998; Ferguson, 1996; Howland, 1972; Van Saun \& Sniffen, 1996). Esses fatos permitem supor que nas vacas em lactação e com anestro por longo período, condição em que os ovários quase sempre sofrem alterações no tamanho (ficam menores) e consistência (duros), provavelmente o tempo de retorno ao cio das mesmas, com o mesmo nível nutricional oferecido no presente trabalho, seria maior que os 114,0 $\pm 37,9$ dias verificados nesse estudo, pois nove das 12 vacas não lactante do grupo tratado apresentavam ovários inativos (acíclicos), mas de tamanho e consistência normais ou próximos do normal.

O número de dias para recuperar a AOLC de vacas mestiça Holandês x Zebu magras com anestro e o ganho de peso médio diário dos animais mostraram uma relação linear negativa (Figura 1), conforme a 
equação $\mathrm{Y}=256,6$-196,2X, onde $\mathrm{X}$ foi o ganho de peso por dia $(\mathrm{kg})$ e $\mathrm{Y}$ o número de dias para recuperar a $\operatorname{AOLC}\left(\mathrm{R}^{2}=0,81\right)$.

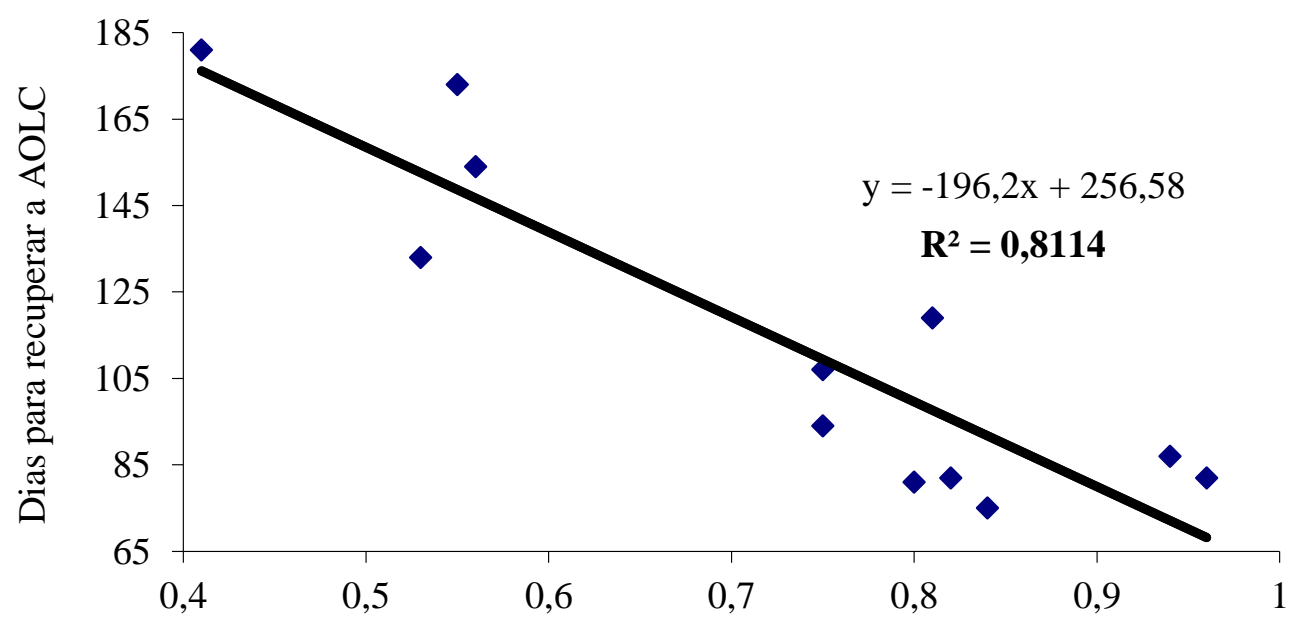

Ganho de Peso / dia (kg/dia)

Figura 1: Tempo (dias) para vacas mestiças leiteiras magras em anestro recuperarem a atividade ovariana luteal cíclica (AOLC), em função do ganho de peso médio diário (kg).

Pode-se citar o seguinte exemplo de aplicação da fórmula obtida na Figura 1: uma vaca Girolanda magra e em anestro, quando alimentada para ganhar $0,500 \mathrm{~g} / \mathrm{dia}(0,5 \mathrm{~kg}) / \mathrm{dia}$, em quantos dias deve apresentar atividade ovariana luteal cíclica? $\mathrm{Y}=256,58-196,2 \mathrm{x}$ ou $\mathrm{Y}=256,58-196,2 \times 0,500$ ou $\mathrm{Y}=256,58-98,1$ ou $\mathrm{Y}=158,5$ dias (resposta).

Para cada dia de atraso na concepção após 40 dias do parto são relatadas perdas de 2,4 $\pm 1,9 \mathrm{~kg}$ de leite/dia em vacas com produção acima de $6.000 \mathrm{~kg}$ leite por lactação (Louca \& Legates, 1968); 0,90 kg de leite/dia na produção anual com período de serviço 20 dias além do desejável (Haresign \& Lewis, 1979); 20 a 25 francos por dia não prenhe acima de três meses pós-parto (Fivaz, 1981), um dólar por cada dia não gestante após 85 dias do parto (Deletang, 1983) e três dólares por dia não gestante além de 60 dias pós-parto (Sowden, 1990). Esses autores enfatizam como o desempenho reprodutivo é de grande importância econômica na atividade leiteira, o que também é afirmado por Esslemont (1992), Gröhn \& Rajala-Schultz (2000) e Roche et al. (2000). Os resultados do presente trabalho mostram como é indesejável permitir que o bovino adquira a condição de anestro por subnutrição, pois a demora na recuperação da AOLC destas vacas em anestro atrasa a concepção e, consequentemente, alonga o período de serviço e o intervalo de partos (menos leite e bezerros), acarretando grandes perdas e onerando o custo de produção (Ferreira \& Teixeira, 2000; Stevenson, 1996a, 1996b).

\section{Conclusão}

A recuperação da atividade ovariana luteal cíclica (volta do cio) em vacas mestiça Holandês x Zebu, não lactante, magras e com anestro por ovários inativos, é um processo demorado (114,0 \pm 37,9 dias) e dependente do ganho de peso médio diário, que no presente trabalho foi de $0,68 \mathrm{~kg} / \mathrm{dia}$.

\section{Referências bibliográficas}

Almeida Neto, J.R.M. Dinâmica ovariana em vacas magras com anestro e taxa de prenhez com IATF em vacas mestiças leiteiras de diferentes escores da condição corporal. Universidade Federal de Viçosa, Departamento de Veterinária, Viçosa-MG, 2013. 51p. (Tese de Doutorado). 
ANUALPEC. (2017). Anuário da Pecuária Brasileira (20th ed. Vol. 1). São Paulo, SP, Brasil: Instituto FNP.

Beam, S. W. \& Butler, W. R. (1999). Effects of energy balance on follicular development and first ovulation in postpartum dairy cows. Journal of Reproduction and Fertility, 54411-424.

Butler, W. R. (1998). Review: Effect of protein nutrition on ovarian and uterine physiology in dairy cattle. Journal of Dairy Science, 81(9):2533-2539.

Deletang, F. (1983). Fecondité, la conduite a tenir. Elevage Bovin, 13044-46.

Drion, P., Beckers, J.-F., Derkenne, F. \& Hanzen, C. (2000). Le développement folliculaire chez la vache. 2. Mécanismes hormonaux au cours du cycle et du postpartum. Annales de Médecine Vétérinaire, 144385-404.

Esslemont, R. J. (1992). Measuring dairy herd fertility. The Veterinary Record, 131(10):209-212.

Ferguson, J. D. (1996). Diet, production and reproduction in dairy cows. Animal Feed Science and Technology, 59(1-3):173-184.

Ferreira, A., Sá, W. M. F., Villaça, H. A. \& Assis, A. G. (1992). Diagnóstico da situação produtiva e reprodutiva em rebanhos bovinos leiteiros da zona da mata de Minas Gerais. Pesquisa Agropecuária Brasileira, 27(1):91-104.

Ferreira, A. M. (2010). Reprodução da fêmea bovina: Fisiologia aplicada e problemas mais comuns (causas e tratamentos) (Vol. 1). Juiz de Fora, Minas Gerais: Editar Editora.

Ferreira, A. M. (2012). Manejo reprodutivo de bovinos leiteiros: Práticas corretas e incorretas, casos reais, perguntas e respostas (Vol. 1). Juiz de Fora, Minas Gerais: Editar Editora.

Ferreira, A. M. \& Teixeira, N. M. (2000). Estimativas de mudança na produção de leite com a variação do intervalo de partos em rebanhos bovinos. Revista Brasileira de Reprodução Animal, 24(4):177-181.

Fivaz, B. H. (1981). Current aspects of the post-partum period of the dairy cow. Zimbabwe Veterinary Journal, 12(2/3):22-26.

Gröhn, Y. T. \& Rajala-Schultz, P. J. (2000). Epidemiology of reproductive performance in dairy cows. Animal Reproduction Science, 60605-614.

Grummer, R. R., Wiltbank, M. C., Fricke, P. M., Watters, R. D. \& Silva-Del-Rio, N. (2010). Management of dry and transition cows to improve energy balance and reproduction. Journal of Reproduction and Development, 56S22-S28.

Gwazdauskas, F. C., Kendrick, K. W., Pryor, A. W. \& Bailey, T. L. (2000). Impact of follicular aspiration on folliculogenesis as influenced by dietary energy and stage of lactation. Journal of Dairy Science, 83(7):1625-1634.

Hale, D. H. (1975). Nutrition, hormones and fertillity. Rhodesian Agriculture Journal, Rhodesian Agriculture Journal(3):69-74.

Haresign, W. \& Lewis, D. (1979). Recent advances in animal nutrition. Boston, USA: Butterworths.

Hess, B. W., Lake, S. L., Scholljegerdes, E. J., Weston, T. R., Nayigihugu, V., Molle, J. D. C. \& Moss, G. E. (2005). Nutritional controls of beef cow reproduction. Journal of Animal Science, 83(Suppl 13):E90E106.

Howland, B. E. (1972). Ovarian weight and ovarian compensatory hypertrophy in the rat as affected by duration of underfeeding. Reproduction, 28(2):321-323.

Imakawa, K., Day, M. L., Garcia-Winder, M., Zalesky, D. D., Kittok, R. J., Schanbacher, B. D. \& Kinder, J. E. (1986). Endocrine changes during restoration of estrous cycles following induction of anestrus by restricted nutrient intake in beef heifers. Journal of Animal Science, 63(2):565-571.

Louca, A. \& Legates, J. E. (1968). Produccion losses in dairy cattle open days. Journal Dairy Science, 51(4):573-583. 
Mapletoft, R. J., Bó, G. A. \& Adams, G. P. (2000). Avanços na manipulação do ciclo estral de doadoras e receptoras nos programas de transferência de embriões em bovinos. Arquivos da Faculdade de Veterinária da UFRGS, 28(1):24-51.

Montiel, F. \& Ahuja, C. (2005). Body condition and suckling as factors influencing the duration of postpartum anestrus in cattle: a review. Animal Reproduction Science, 85(1-2):1-26.

Pereira, E. S., Pimentel, P. G., Queiroz, A. C. \& Mizubuti, I. Y. (2010). Novilhas leiteiras (Vol. 1). Fortaleza, Ceará: Graphiti Gráfica e Editora Ltda.

Peter, A. T., Vos, P. L. A. M. \& Ambrose, D. J. (2009). Postpartum anestrus in dairy cattle. Theriogenology, 71(9):1333-1342.

Rhodes, F. M., Fitzpatrick, L. A., Entwistle, K. W. \& De'Ath, G. (1995). Sequential changes in ovarian follicular dynamics in Bos indicus heifers before and after nutritional anoestrus. Reproduction, 104(1):41-49.

Richards, M. W., Wettemann, R. P. \& Schoenemann, H. M. (1986). Effect of restricted intake on cyclic ovarian activity and estrus in Hereford cows. Animal Science Research Report, 118308-311.

Roche, J. F., Mackey, D. \& Diskin, M. D. (2000). Reproductive management of postpartum cows. Animal Reproduction Science, 60703-712.

Schillo, K. K. (1992). Effects of dietary energy on control of luteinizing hormone secretion in cattle and sheep. journal of Animal Science, 70(4):1271-1282.

Short, R. E., Bellows, R. A., Staigmiller, R. B., Berardinelli, J. G. \& Custer, E. E. (1990). Physiological mechanisms controlling anestrus and infertility in postpartum beef cattle. Journal of Animal Science, 68(3):799-816.

Sowden, C. L. (1990). Culling economics. Dairy Herd Management, 27(6):22-26.

Stagg, K., Spicer, L. J., Sreenan, J. M., Roche, J. F. \& Diskin, M. G. (1998). Effect of calf isolation on follicular wave dynamics, gonadotropin and metabolic hormone changes, and interval to first ovulation in beef cows fed either of two energy levels postpartum. Biology of Reproduction, 59(4):777-783.

Stevenson, J. (1996a). Dissecting a calving interval. Hoard's Dairyman, Atkinson, 141326.

Stevenson, J. (1996b). Is there an optimal calving interval. Hoard's Dairyman, 141408.

Tervit, H. R., Smith, J. F. \& Kaltenbach, C. C. (1977). Post-partum anoestrus in beef cattle: a review. Proceedings of the New Zealand Society of Animal Production (New Zealand), 37109-119.

Van Saun, R. J. \& Sniffen, C. J. (1996). Nutritional management of the pregnant dairy cow to optimize health, lactation and reproductive performance. Animal Feed Science and Technology, 59(1-3):13-26.

Vanholder, T., Leroy, J. L. M. R., Van Soom, A., Opsomer, G., Maes, D., Coryn, M. \& Kruif, A. (2005). Effect of non-esterified fatty acids on bovine granulosa cell steroidogenesis and proliferation in vitro. Animal Reproduction Science, 87(1-2):33-44.

Wiltbank, M. C., Gallagher, K. P., Christensen, A. K., Brabec, R. K. \& Keyes, P. L. (1990). Physiological and immunocytochemical evidence for a new concept of blood flow regulation in the corpus luteum. Biology of Reproduction, 42(1):139-149.

Wiltbank, M. C., Gümen, A. \& Sartori, R. (2002). Physiological classification of anovulatory conditions in cattle. Theriogenology, 57(1):21-52.

Recebido: 11 de janeiro, 2019

Aprovado: 5 fevereiro, 2019.

Publicado: 26 fevereiro, 2019.

Licenciamento: Este artigo é publicado na modalidade Acesso Aberto sob a licença Creative Commons Atribuição 4.0 (CC-BY 4.0), a qual permite uso irrestrito, distribuição, reprodução em qualquer meio, desde que o autor e a fonte sejam devidamente creditados. 